PROCEEDINGS OF THE

AMERICAN MATHEMATICAL SOCIETY

Volume 143, Number 4, April 2015, Pages 1605-1615

S 0002-9939(2014)12340-9

Article electronically published on December 19, 2014

\title{
ON BOUNDARY HÖLDER GRADIENT ESTIMATES FOR SOLUTIONS TO THE LINEARIZED MONGE-AMPÈRE EQUATIONS
}

\author{
NAM Q. LE AND OVIDIU SAVIN \\ (Communicated by Joachim Krieger)
}

\begin{abstract}
In this paper, we establish boundary Hölder gradient estimates for solutions to the linearized Monge-Ampère equations with $L^{p}(n<p \leq \infty)$ right-hand side and $C^{1, \gamma}$ boundary values under natural assumptions on the domain, boundary data and the Monge-Ampère measure. These estimates extend our previous boundary regularity results for solutions to the linearized Monge-Ampère equations with bounded right-hand side and $C^{1,1}$ boundary data.
\end{abstract}

\section{Statement of the Main Results}

In this paper, we establish boundary Hölder gradient estimates for solutions to the linearized Monge-Ampère equations with $L^{p}(n<p \leq \infty)$ right-hand side and $C^{1, \gamma}$ boundary values under natural assumptions on the domain, boundary data and the Monge-Ampère measure. Before stating these estimates, we introduce the following assumptions on the domain $\Omega$ and function $\phi$.

Let $\Omega \subset \mathbb{R}^{n}$ be a bounded convex set with

$$
B_{\rho}\left(\rho e_{n}\right) \subset \Omega \subset\left\{x_{n} \geq 0\right\} \cap B_{\frac{1}{\rho}},
$$

for some small $\rho>0$. Assume that

$\Omega$ contains an interior ball of radius $\rho$ tangent to $\partial \Omega$ at each point on $\partial \Omega \cap B_{\rho}$.

Let $\phi: \bar{\Omega} \rightarrow \mathbb{R}, \phi \in C^{0,1}(\bar{\Omega}) \cap C^{2}(\Omega)$ be a convex function satisfying

$$
0<\lambda \leq \operatorname{det} D^{2} \phi \leq \Lambda \text { in } \Omega \text {. }
$$

Throughout, we denote by $\Phi=\left(\Phi^{i j}\right)$ the matrix of cofactors of the Hessian matrix $D^{2} \phi$, i.e.,

$$
\Phi=\left(\operatorname{det} D^{2} \phi\right)\left(D^{2} \phi\right)^{-1} .
$$

We assume that on $\partial \Omega \cap B_{\rho}$, $\phi$ separates quadratically from its tangent planes on $\partial \Omega$. Precisely we assume that if $x_{0} \in \partial \Omega \cap B_{\rho}$, then

$$
\rho\left|x-x_{0}\right|^{2} \leq \phi(x)-\phi\left(x_{0}\right)-\nabla \phi\left(x_{0}\right)\left(x-x_{0}\right) \leq \rho^{-1}\left|x-x_{0}\right|^{2},
$$

for all $x \in \partial \Omega$.

Received by the editors May 17, 2013 and, in revised form, August 8, 2013.

2010 Mathematics Subject Classification. Primary 35J70, 35B65, 35B45, 35J96.

Key words and phrases. Linearized Monge-Ampère equations, localization theorem, boundary gradient estimates. 
Let $S_{\phi}\left(x_{0}, h\right)$ be the section of $\phi$ centered at $x_{0} \in \bar{\Omega}$ and of height $h$ :

$$
S_{\phi}\left(x_{0}, h\right):=\left\{x \in \bar{\Omega}: \phi(x)<\phi\left(x_{0}\right)+\nabla \phi\left(x_{0}\right)\left(x-x_{0}\right)+h\right\} .
$$

When $x_{0}$ is the origin, we denote for simplicity $S_{h}:=S_{\phi}(0, h)$.

Now, we can state our boundary Hölder gradient estimates for solutions to the linearized Monge-Ampère equations with $L^{p}$ right-hand side and $C^{1, \gamma}$ boundary data.

Theorem 1.1. Assume $\phi$ and $\Omega$ satisfy the assumptions (1.1)-(1.4) above. Let $u: B_{\rho} \cap \bar{\Omega} \rightarrow \mathbb{R}$ be a continuous solution to

$$
\left\{\begin{aligned}
\Phi^{i j} u_{i j} & =f \text { in } B_{\rho} \cap \Omega, \\
u & =\varphi \text { on } \partial \Omega \cap B_{\rho},
\end{aligned}\right.
$$

where $f \in L^{p}\left(B_{\rho} \cap \Omega\right)$ for some $p>n$ and $\varphi \in C^{1, \gamma}\left(B_{\rho} \cap \partial \Omega\right)$. Then, there exist $\alpha \in(0,1)$ and $\theta_{0}$ small depending only on $n, p, \rho, \lambda, \Lambda, \gamma$ such that for all $\theta \leq \theta_{0}$ we have

$$
\begin{aligned}
& \|u-u(0)-\nabla u(0) x\|_{L^{\infty}\left(S_{\theta}\right)} \\
& \quad \leq C\left(\|u\|_{L^{\infty}\left(B_{\rho} \cap \Omega\right)}+\|f\|_{L^{p}\left(B_{\rho} \cap \Omega\right)}+\|\varphi\|_{C^{1, \gamma}\left(B_{\rho} \cap \partial \Omega\right)}\right)\left(\theta^{1 / 2}\right)^{1+\alpha}
\end{aligned}
$$

where $C$ depends only on $n, p, \rho, \lambda, \Lambda, \gamma$. We can take $\alpha:=\min \left\{1-\frac{n}{p}, \gamma\right\}$ provided that $\alpha<\alpha_{0}$ where $\alpha_{0}$ is the exponent in our previous boundary Hölder gradient estimates (see Theorem 2.11).

Theorem 1.1 extends our previous boundary Hölder gradient estimates for solutions to the linearized Monge-Ampère equations with bounded right-hand side and $C^{1,1}$ boundary data [5, Theorem 2. 1]. This is an affine invariant analogue of the boundary Hölder gradient estimates of Ural'tseva [9] (see also [10] for a survey) for uniformly elliptic equation with $L^{p}$ right-hand side.

Remark 1.2. By the Localization Theorem [6,7, we have

$$
B_{c \theta^{1 / 2} /|\log \theta|} \cap \bar{\Omega} \subset S_{\theta} \subset B_{C \theta^{1 / 2}|\log \theta|} \cap \bar{\Omega} .
$$

Therefore, Theorem 1.1 easily implies that $\nabla u$ is $C^{0, \alpha^{\prime}}$ on $B_{\rho / 2} \cap \partial \Omega$ for all $\alpha^{\prime}<\alpha$.

As a consequence of Theorem 1.1, we obtain global $C^{1, \alpha}$ estimates for solutions to the linearized Monge-Ampère equations with $L^{p}(n<p \leq \infty)$ right-hand side and $C^{1, \gamma}$ boundary values under natural assumptions on the domain, boundary data and continuity of the Monge-Ampère measure.

Theorem 1.3. Assume that $\Omega \subset B_{1 / \rho}$ contains an interior ball of radius $\rho$ tangent to $\partial \Omega$ at each point on $\partial \Omega$. Let $\phi: \bar{\Omega} \rightarrow \mathbb{R}, \phi \in C^{0,1}(\bar{\Omega}) \cap C^{2}(\Omega)$ be a convex function satisfying

$$
\operatorname{det} D^{2} \phi=g \quad \text { with } \quad \lambda \leq g \leq \Lambda, \quad g \in C(\bar{\Omega}) .
$$

Assume further that on $\partial \Omega$, $\phi$ separates quadratically from its tangent planes, namely

$$
\rho\left|x-x_{0}\right|^{2} \leq \phi(x)-\phi\left(x_{0}\right)-\nabla \phi\left(x_{0}\right)\left(x-x_{0}\right) \leq \rho^{-1}\left|x-x_{0}\right|^{2}, \forall x, x_{0} \in \partial \Omega .
$$


Let $u: \bar{\Omega} \rightarrow \mathbb{R}$ be a continuous function that solves the linearized Monge-Ampère equation

$$
\left\{\begin{aligned}
\Phi^{i j} u_{i j} & =f \text { in } \Omega, \\
u & =\varphi \text { on } \partial \Omega,
\end{aligned}\right.
$$

where $\varphi$ is a $C^{1, \gamma}$ function defined on $\partial \Omega(0<\gamma \leq 1)$ and $f \in L^{p}(\Omega)$ with $p>n$. Then

$$
\|u\|_{C^{1, \beta}(\bar{\Omega})} \leq K\left(\|\varphi\|_{C^{1, \gamma}(\partial \Omega)}+\|f\|_{L^{p}(\Omega)}\right)
$$

where $\beta \in(0,1)$ and $K$ are constants depending on $n, \rho, \gamma, \lambda, \Lambda, p$ and the modulus of continuity of $g$.

Theorem 1.3 extends our previous global $C^{1, \alpha}$ estimates for solutions to the linearized Monge-Ampère equations with bounded right-hand side and $C^{1,1}$ boundary data [5, Theorem 2. 5 and Remark 7.1]. It is also the global counterpart of Gutiérrez-Nguyen's interior $C^{1, \alpha}$ estimates for the linearized Monge-Ampère equations. If we assume $\varphi$ to be more regular, say $\varphi \in W^{2, q}(\Omega)$ where $q>p$, then Theorem 1.3 is a consequence of the global $W^{2, p}$ estimates for solutions to the linearized Monge-Ampère equations [4, Theorem 1. 2]. In this case, the proof in [4] is quite involved. Our proof of Theorem 1.3 here is much simpler.

Remark 1.4. The estimates in Theorem 1.3 can be improved to

$$
\|u\|_{C^{1, \beta}(\bar{\Omega})} \leq K\left(\|\varphi\|_{C^{1, \gamma}(\partial \Omega)}+\|f / \operatorname{tr} \Phi\|_{L^{p}(\Omega)}\right) .
$$

This follows easily from the estimates in Theorem 1.3 and the global $W^{2, p}$ estimates for solutions to the standard Monge-Ampère equations with continuous right-hand side [8]. Indeed, since

$$
\operatorname{tr} \Phi \geq n(\operatorname{det} \Phi)^{\frac{1}{n}} \geq n \lambda^{\frac{n-1}{n}},
$$

we also have $f / \operatorname{tr} \Phi \in L^{p}(\Omega)$. Fix $q \in(n, p)$, then by [8], $\operatorname{tr} \Phi \in L^{\frac{p q}{p-q}}(\Omega)$. Now apply the estimates in Theorem 1.3 to $f \in L^{q}(\Omega)$ and then use Hölder inequality to $f=(f / \operatorname{tr} \Phi)(\operatorname{tr} \Phi)$ to obtain (1.5).

Remark 1.5. The linearized Monge-Ampère operator $L_{\phi}:=\Phi^{i j} \partial_{i j}$ with $\phi$ satisfying the conditions of Theorem 1.3 is in general degenerate. Here is an explicit example in two dimensions, taken from [11], showing that $L_{\phi}$ is not uniformly elliptic in $\bar{\Omega}$. Consider

$$
\phi(x, y)=\frac{x^{2}}{\log \left|\log \left(x^{2}+y^{2}\right)\right|}+y^{2} \log \left|\log \left(x^{2}+y^{2}\right)\right|
$$

in a small ball $\Omega=B_{\rho}(0) \subset R^{2}$ around the origin. Then $\phi \in C^{0,1}(\bar{\Omega}) \cap C^{2}(\Omega \backslash\{0\})$ is strictly convex with

$$
\operatorname{det} D^{2} \phi(x, y)=4+O\left(\frac{\log \left|\log \left(x^{2}+y^{2}\right)\right|}{\log \left(x^{2}+y^{2}\right)}\right) \in C(\bar{\Omega})
$$

and $\phi$ has smooth boundary data on $\partial \Omega$. The quadratic separation of $\phi$ from its tangent planes on $\partial \Omega$ can be readily checked (see also [7, Proposition 3.2]). However $\phi \notin W^{2, \infty}(\Omega)$.

Remark 1.6. For the global $C^{1, \alpha}$ estimates in Theorem 1.3, the condition $p>n$ is sharp, since even in the uniformly elliptic case (for example, when $\phi(x)=\frac{1}{2}|x|^{2}$, $L_{\phi}$ is the Laplacian), the global $C^{1, \alpha}$ estimates fail when $p=n$. 
We prove Theorem 1.1 using the perturbation arguments in the spirit of Caffarelli [1,2] (see also Wang [12]) in combination with our previous boundary Hölder gradient estimates for the case of bounded right-hand side $f$ and $C^{1,1}$ boundary data $[5$.

The next section will provide the proof of Theorem 1.1. The proof of Theorem 1.3 will be given in the final section, Section 3 .

\section{Boundary Hölder GRAdient estimates}

In this section, we prove Theorem 1.1. We will use the letters $c, C$ to denote generic constants depending only on the structural constants $n, p, \rho, \gamma, \lambda, \Lambda$ that may change from line to line.

Assume $\phi$ and $\Omega$ satisfy the assumptions (1.1)-(1.4). We can also assume that $\phi(0)=0$ and $\nabla \phi(0)=0$. By the Localization Theorem for solutions to the MongeAmpère equations proved in [6,7], there exists a small constant $k$ depending only on $n, \rho, \lambda, \Lambda$ such that if $h \leq k$ then

$$
k E_{h} \cap \bar{\Omega} \subset S_{\phi}(0, h) \subset k^{-1} E_{h} \cap \bar{\Omega}
$$

where

$$
E_{h}:=h^{1 / 2} A_{h}^{-1} B_{1}
$$

with $A_{h}$ being a linear transformation (sliding along the $x_{n}=0$ plane)

$$
A_{h}(x)=x-\tau_{h} x_{n}, \tau_{h} \cdot e_{n}=0, \operatorname{det} A_{h}=1
$$

and

$$
\left|\tau_{h}\right| \leq k^{-1}|\log h| .
$$

We define the following rescaling of $\phi$

$$
\phi_{h}(x):=\frac{\phi\left(h^{1 / 2} A_{h}^{-1} x\right)}{h}
$$

in

$$
\Omega_{h}:=h^{-1 / 2} A_{h} \Omega .
$$

Then

$$
\lambda \leq \operatorname{det} D^{2} \phi_{h}(x)=\operatorname{det} D^{2} \phi\left(h^{1 / 2} A_{h}^{-1} x\right) \leq \Lambda
$$

and

$$
B_{k} \cap \overline{\Omega_{h}} \subset S_{\phi_{h}}(0,1)=h^{-1 / 2} A_{h} S_{h} \subset B_{k^{-1}} \cap \overline{\Omega_{h}} .
$$

Lemma 4.2 in [5] implies that if $h, r \leq c$ small then $\phi_{h}$ satisfies in $S_{\phi_{h}}(0,1)$ the hypotheses of the Localization Theorem [6, 7] at all $x_{0} \in S_{\phi_{h}}(0, r) \cap \partial S_{\phi_{h}}(0,1)$. In particular, there exists $\tilde{\rho}$ small, depending only on $n, \rho, \lambda, \Lambda$ such that if $x_{0} \in$ $S_{\phi_{h}}(0, r) \cap \partial S_{\phi_{h}}(0,1)$ then

$$
\tilde{\rho}\left|x-x_{0}\right|^{2} \leq \phi_{h}(x)-\phi_{h}\left(x_{0}\right)-\nabla \phi_{h}\left(x_{0}\right)\left(x-x_{0}\right) \leq \tilde{\rho}^{-1}\left|x-x_{0}\right|^{2},
$$

for all $x \in \partial S_{\phi_{h}}(0,1)$. We fix $r$ in what follows.

Our previous boundary Hölder gradient estimates [5] for solutions to the linearized Monge-Ampère with bounded right-hand side and $C^{1,1}$ boundary data hold in $S_{\phi_{h}}(0, r)$. They will play a crucial role in the perturbation arguments and we now recall them here. 
Theorem 2.1 ([5, Theorem 2.1 and Proposition 6.1]). Assume $\phi$ and $\Omega$ satisfy the assumptions (1.1)-(1.4) above. Let $u: S_{r} \cap \bar{\Omega} \rightarrow \mathbb{R}$ be a continuous solution to

$$
\left\{\begin{aligned}
\Phi^{i j} u_{i j} & =f \text { in } S_{r} \cap \Omega, \\
u & =0 \text { on } \partial \Omega \cap S_{r},
\end{aligned}\right.
$$

where $f \in L^{\infty}\left(S_{r} \cap \Omega\right)$. Then

$$
\left|\partial_{n} u(0)\right| \leq C_{0}\left(\|u\|_{L^{\infty}\left(S_{r} \cap \Omega\right)}+\|f\|_{L^{\infty}\left(S_{r} \cap \Omega\right)}\right)
$$

and for $s \leq r / 2$

$$
\max _{S_{r}}\left|u-\partial_{n} u(0) x_{n}\right| \leq C_{0}\left(s^{1 / 2}\right)^{1+\alpha_{0}}\left(\|u\|_{L^{\infty}\left(S_{r} \cap \Omega\right)}+\|f\|_{L^{\infty}\left(S_{r} \cap \Omega\right)}\right)
$$

where $\alpha_{0} \in(0,1)$ and $C_{0}$ are constants depending only on $n, \rho, \lambda, \Lambda$.

Now, we are ready to give the proof of Theorem 1.1 .

Proof of Theorem 1.1. Since $\left.u\right|_{\partial \Omega \cap B_{\rho}}$ is $C^{1, \gamma}$, by subtracting a suitable linear function we can assume that on $\partial \Omega \cap B_{\rho}, u$ satisfies

$$
|u(x)| \leq M\left|x^{\prime}\right|^{1+\gamma} .
$$

Let

$$
\alpha:=\min \left\{\gamma, 1-\frac{n}{p}\right\}
$$

if $\alpha<\alpha_{0}$; otherwise let $\alpha \in\left(0, \alpha_{0}\right)$ where $\alpha_{0}$ is in Theorem 2.1. The only place where we need $\alpha<\alpha_{0}$ is (2.12).

By dividing our equation by a suitable constant we may assume that for some $\theta$ to be chosen later

$$
\|u\|_{L^{\infty}\left(B_{\rho} \cap \Omega\right)}+\|f\|_{L^{p}\left(B_{\rho} \cap \Omega\right)}+M \leq\left(\theta^{1 / 2}\right)^{1+\alpha}=: \delta .
$$

Claim. There exists $0<\theta_{0}<r / 4$ small depending only on $n, \rho, \lambda, \Lambda, \gamma, p$, and a sequence of linear functions

$$
l_{m}(x):=b_{m} x_{n}
$$

with $b_{0}=b_{1}=0$ such that for all $\theta \leq \theta_{0}$ and for all $m \geq 1$, we have

$$
\left\|u-l_{m}\right\|_{L^{\infty}\left(S_{\theta} m\right)} \leq\left(\theta^{m / 2}\right)^{1+\alpha},
$$

and

$$
\left|b_{m}-b_{m-1}\right| \leq C_{0}\left(\theta^{\frac{m-1}{2}}\right)^{\alpha}
$$

Our theorem follows from the claim. Indeed, (ii) implies that $\left\{l_{m}\right\}$ converges uniformly in $S_{\theta}$ to a linear function $l(x)=b x_{n}$ with $b$ universally bounded since

$$
|b| \leq \sum_{m=1}^{\infty}\left|b_{m}-b_{m-1}\right| \leq \sum_{m=1}^{\infty} C_{0}\left(\theta^{\theta / 2}\right)^{m-1}=\frac{C_{0}}{1-\theta^{\alpha / 2}} \leq 2 C_{0} .
$$


Furthermore, by (2.6) and (2.7), we have $\left|x_{n}\right| \leq k^{-1} \theta^{m / 2}$ for $x \in S_{\theta^{m}}$. Therefore, for any $m \geq 1$,

$$
\begin{aligned}
\|u-l\|_{L^{\infty}\left(S_{\theta^{m}}\right)} & \leq\left\|u-l_{m}\right\|_{L^{\infty}\left(S_{\theta^{m}}\right)}+\sum_{j=m+1}^{\infty}\left\|l_{j}-l_{j-1}\right\|_{L^{\infty}\left(S_{\theta^{m}}\right)} \\
& \leq\left(\theta^{m / 2}\right)^{1+\alpha}+\sum_{j=m+1}^{\infty} C_{0}\left(\theta^{\frac{j-1}{2}}\right)^{\alpha}\left(k^{-1} \theta^{m / 2}\right) \\
& \leq C\left(\theta^{m / 2}\right)^{1+\alpha} .
\end{aligned}
$$

We now prove the claim by induction. Clearly (i) and (ii) hold for $m=1$. Suppose (i) and (ii) hold up to $m \geq 1$. We prove them for $m+1$. Let $h=\theta^{m}$. We define the rescaled domain $\Omega_{h}$ and function $\phi_{h}$ as in (2.9) and (2.8). We also define for $x \in \Omega_{h}$

$$
v(x):=\frac{\left(u-l_{m}\right)\left(h^{1 / 2} A_{h}^{-1} x\right)}{h^{\frac{1+\alpha}{2}}}, f_{h}(x):=h^{\frac{1-\alpha}{2}} f\left(h^{1 / 2} A_{h}^{-1} x\right) .
$$

Then

$$
\|v\|_{L^{\infty}\left(S_{\phi_{h}}(0,1)\right)}=\frac{1}{h^{\frac{1+\alpha}{2}}}\left\|u-l_{m}\right\|_{L^{\infty}\left(S_{h}\right)} \leq 1
$$

and

$$
\Phi_{h}^{i j} v_{i j}=f_{h} \text { in } S_{\phi_{h}}(0,1)
$$

with

$$
\left\|f_{h}\right\|_{L^{p}\left(S_{\phi_{h}}(0,1)\right)}=\left(h^{1 / 2}\right)^{1-\alpha-n / p}\|f\|_{L^{p}\left(S_{h}\right)} \leq \delta .
$$

Let $w$ be the solution to

$$
\left\{\begin{aligned}
\Phi_{h}^{i j} w_{i j} & =0 \quad \text { in } S_{\phi_{h}}(0,2 \theta), \\
w & =\varphi_{h} \text { on } \partial S_{\phi_{h}}(0,2 \theta),
\end{aligned}\right.
$$

where

$$
\varphi_{h}= \begin{cases}0 & \text { on } \partial S_{\phi_{h}}(0,2 \theta) \cap \partial \Omega_{h} \\ v & \text { on } \partial S_{\phi_{h}}(0,2 \theta) \cap \Omega_{h} .\end{cases}
$$

By the maximum principle, we have

$$
\|w\|_{L^{\infty}\left(S_{\phi_{h}}(0,2 \theta)\right)} \leq\|v\|_{L^{\infty}\left(S_{\phi_{h}}(0,2 \theta)\right)} \leq 1 .
$$

Let

$$
\bar{l}(x):=\bar{b} x_{n} ; \bar{b}:=\partial_{n} w(0) .
$$

Then the boundary Hölder gradient estimates in Theorem 2.1 give

$$
|\bar{b}| \leq C_{0}\|w\|_{L^{\infty}\left(S_{\phi_{h}}(0,2 \theta)\right)} \leq C_{0}
$$

and

$$
\begin{aligned}
\|w-\bar{l}\|_{L^{\infty}\left(S_{\phi_{h}}(0, \theta)\right)} \leq C_{0}\|w\|_{L^{\infty}\left(S_{\phi_{h}}(0,2 \theta)\right)}\left(\theta^{\frac{1}{2}}\right)^{1+\alpha_{0}} & \leq C_{0}\left(\theta^{\frac{1}{2}}\right)^{1+\alpha_{0}} \\
& \leq \frac{1}{2}\left(\theta^{\frac{1}{2}}\right)^{1+\alpha}
\end{aligned}
$$

provided that

$$
C_{0} \theta_{0}^{\frac{\alpha_{0}-\alpha}{2}} \leq 1 / 2
$$

We will show that, by choosing $\theta \leq \theta_{0}$ where $\theta_{0}$ is small, we have

$$
\|w-v\|_{L^{\infty}\left(S_{\phi_{h}}(0,2 \theta)\right)} \leq \frac{1}{2}\left(\theta^{\frac{1}{2}}\right)^{1+\alpha} .
$$


Combining this with (2.12), we obtain

$$
\|v-\bar{l}\|_{L^{\infty}\left(S_{\phi_{h}}(0, \theta)\right)} \leq\left(\theta^{\frac{1}{2}}\right)^{1+\alpha} .
$$

Now, let

$$
l_{m+1}(x):=l_{m}(x)+\left(h^{1 / 2}\right)^{1+\alpha} \bar{l}\left(h^{-1 / 2} A_{h} x\right) .
$$

Then, for $x \in S_{\theta^{m+1}}=S_{\theta h}$, we have $h^{-1 / 2} A_{h} x \in S_{\phi_{h}}(0, \theta)$ and

$\left(u-l_{m+1}\right)(x)=u(x)-l_{m}(x)-\left(h^{1 / 2}\right)^{1+\alpha} \bar{l}\left(h^{-1 / 2} A_{h} x\right)=\left(h^{1 / 2}\right)^{1+\alpha}(v-\bar{l})\left(h^{-1 / 2} A_{h} x\right)$.

Thus

$$
\begin{aligned}
\left\|u-l_{m+1}\right\|_{L^{\infty}\left(S_{\theta^{m+1}}\right)}=\left(h^{1 / 2}\right)^{1+\alpha}\|v-\bar{l}\|_{L^{\infty}\left(S_{\phi_{h}}(0, \theta)\right)} & \\
& \leq\left(h^{1 / 2}\right)^{1+\alpha}\left(\theta^{1 / 2}\right)^{1+\alpha}=\left(\theta^{\frac{m+1}{2}}\right)^{1+\alpha},
\end{aligned}
$$

proving (i). On the other hand, we have

$$
l_{m+1}(x)=b_{m+1} x_{n}
$$

where, by (2.7)

$$
b_{m+1}:=b_{m}+\left(h^{1 / 2}\right)^{1+\alpha} h^{-1 / 2} \bar{b}=b_{m}+h^{\alpha / 2} \bar{b} .
$$

Therefore, the claim is established since (ii) follows from (2.11) and

$$
\left|b_{m+1}-b_{m}\right|=h^{\alpha / 2}|\bar{b}| \leq C_{0} \theta^{m \alpha / 2} .
$$

It remains to prove (2.13). We will use the $\mathrm{ABP}$ estimate to $w-v$ which solves

$$
\left\{\begin{aligned}
\Phi_{h}^{i j}(w-v)_{i j} & =-f_{h} \quad \text { in } S_{\phi_{h}}(0,2 \theta), \\
w-v & =\varphi_{h}-v \text { on } \partial S_{\phi_{h}}(0,2 \theta) .
\end{aligned}\right.
$$

By this estimate and the way $\varphi_{h}$ is defined, we have

$$
\begin{aligned}
& \|w-v\|_{L^{\infty}\left(S_{\phi_{h}}(0,2 \theta)\right)} \leq\|v\|_{L^{\infty}\left(\partial S_{\phi_{h}}(0,2 \theta) \cap \partial \Omega_{h}\right)} \\
& +C(n) \operatorname{diam}\left(S_{\phi_{h}}(0,2 \theta)\right)\left\|\frac{f_{h}}{\left(\operatorname{det} \Phi_{h}\right)^{\frac{1}{n}}}\right\|_{L^{n}\left(S_{\phi_{h}}(0,2 \theta)\right)} \\
& =:(I)+(I I) .
\end{aligned}
$$

To estimate (I), we denote $y=h^{1 / 2} A_{h}^{-1} x$ when $x \in \partial S_{\phi_{h}}(0,2 \theta) \cap \partial \Omega_{h}$. Then $y \in \partial S_{\phi}(0,2 \theta) \cap \partial \Omega$ and moreover,

$$
y_{n}=h^{1 / 2} x_{n}, y^{\prime}-\nu_{h} y_{n}=h^{1 / 2} x^{\prime} .
$$

Noting that $x \in \partial S_{\phi_{h}}(0,1) \cap \partial \Omega_{h} \subset B_{k^{-1}}$, we have by (2.7)

$$
|y| \leq k^{-1} h^{1 / 2}|\log h||x| \leq h^{1 / 4} \leq \rho
$$

if $h=\theta^{m}$ is small. This is clearly satisfied when $\theta_{0}$ is small.

Since $\Omega$ has an interior tangent ball of radius $\rho$, we have

$$
\left|y_{n}\right| \leq \rho^{-1}\left|y^{\prime}\right|^{2} \text {. }
$$

Therefore

$$
\left|v_{h} y_{n}\right| \leq k^{-1}|\log h| \rho^{-1}\left|y^{\prime}\right|^{2} \leq k^{-1} \rho^{-1} h^{1 / 4}|\log h|\left|y^{\prime}\right| \leq \frac{1}{2}\left|y^{\prime}\right|
$$

and consequently,

$$
\frac{1}{2}\left|y^{\prime}\right| \leq\left|h^{1 / 2} x^{\prime}\right| \leq \frac{3}{2}\left|y^{\prime}\right|
$$


From (2.10)

$$
\tilde{\rho}\left|x^{\prime}\right|^{2} \leq \phi_{h}(x) \leq 2 \theta,
$$

we have

$$
\left|y^{\prime}\right| \leq 2 h^{1 / 2}\left|x^{\prime}\right| \leq 2\left(2 \tilde{\rho}^{-1}\right)^{1 / 2}(\theta h)^{1 / 2} .
$$

By (ii) and $b_{0}=0$, we have

$$
\left|b_{m}\right| \leq \sum_{j=1}^{m}\left|b_{j}-b_{j-1}\right| \leq \sum_{j=1}^{\infty} C_{0}\left(\theta^{\theta / 2}\right)^{j-1}=\frac{C_{0}}{1-\theta^{\alpha / 2}} \leq 2 C_{0}
$$

if

$$
\theta_{0}^{\alpha / 2} \leq 1 / 2
$$

Now, we obtain from the definition of $v$ that

$$
\begin{aligned}
h^{\frac{1+\alpha}{2}}|v(x)|=\mid\left(u-l_{m}\right) & (y)|\leq| u(y)\left|+2 C_{0}\right| y_{n} \mid \\
& \leq \delta\left|y^{\prime}\right|^{1+\gamma}+2 C_{0} \rho^{-1}\left|y^{\prime}\right|^{2}=\left|y^{\prime}\right|^{1+\gamma}\left(\delta+2 C_{0} \rho^{-1}\left|y^{\prime}\right|^{1-\gamma}\right) .
\end{aligned}
$$

Using $\left|y^{\prime}\right| \leq C \theta^{1 / 2}$ and $\gamma \geq \alpha$, we find

$$
\begin{aligned}
& v(x) \leq \frac{C\left((\theta h)^{1 / 2}\right)^{1+\gamma}\left(\delta+\theta^{\frac{1-\gamma}{2}}\right)}{h^{\frac{1+\alpha}{2}}} \\
&=C h^{\gamma-\alpha} \theta^{\frac{1+\gamma}{2}}\left(\theta^{\frac{1+\alpha}{2}}+\theta^{\frac{1-\gamma}{2}}\right) \leq C h^{\gamma-\alpha} \theta \leq \frac{1}{4}\left(\theta^{1 / 2}\right)^{1+\alpha}
\end{aligned}
$$

if $\theta_{0}$ is small. We then obtain

$$
(I) \leq \frac{1}{4}\left(\theta^{1 / 2}\right)^{1+\alpha} .
$$

To estimate (II), we recall $\delta=\left(\theta^{1 / 2}\right)^{1+\alpha}$ and

$$
S_{\phi_{h}}(0,2 \theta) \subset B_{C(2 \theta)^{1 / 2}|\log 2 \theta|} ;\left|S_{\phi_{h}}(0,2 \theta)\right| \leq C(2 \theta)^{n / 2} .
$$

Since

$$
\operatorname{det} \Phi_{h}=\left(\operatorname{det} D^{2} \phi_{h}\right)^{n-1} \geq \lambda^{n-1},
$$

we therefore obtain from Hölder inequality that

$$
\begin{aligned}
(I I) & \leq \frac{C(n)}{\lambda^{\frac{n-1}{n}}} \operatorname{diam}\left(S_{\phi_{h}}(0,2 \theta)\right)\left\|f_{h}\right\|_{L^{n}\left(S_{\phi_{h}}(0,2 \theta)\right)} \\
& \leq C(n, \lambda) \operatorname{diam}\left(S_{\phi_{h}}(0,2 \theta)\right)\left|S_{\phi_{h}}(0,2 \theta)\right|^{\frac{1}{n}-\frac{1}{p}}\left\|f_{h}\right\|_{L^{p}\left(S_{\phi_{h}}(0,2 \theta)\right)} \\
& \leq C \delta \theta^{1 / 2}|\log 2 \theta|\left(\theta^{1 / 2}\right)^{1-n / p}=C\left(\theta^{1 / 2}\right)^{1+\alpha}|\log 2 \theta|\left(\theta^{1 / 2}\right)^{2-n / p} \leq \frac{1}{4}\left(\theta^{1 / 2}\right)^{1+\alpha}
\end{aligned}
$$

if $\theta_{0}$ is small. It follows that

$$
\|w-v\|_{L^{\infty}\left(S_{\phi_{h}}(0,2 \theta)\right)} \leq(I)+(I I) \leq \frac{1}{2}\left(\theta^{\frac{1}{2}}\right)^{1+\alpha},
$$

proving (2.13). The proof of our theorem is complete. 


\section{Global $C^{1, \alpha}$ estimates}

In this section, we will prove Theorem 1.3 ,

Proof of Theorem 1.3. We extend $\varphi$ to a $C^{1, \gamma}(\bar{\Omega})$ function in $\bar{\Omega}$. By the ABP estimate, we have

$$
\|u\|_{L^{\infty}(\Omega)} \leq C\left(\|f\|_{L^{p}(\Omega)}+\|\varphi\|_{L^{\infty}(\bar{\Omega})}\right)
$$

for some $C$ depending on $n, p, \rho, \lambda$. By multiplying $u$ by a suitable constant, we can assume that

$$
\|f\|_{L^{p}(\Omega)}+\|\varphi\|_{C^{1, \gamma}(\bar{\Omega})}=1 .
$$

By using Gutiérrez-Nguyen's interior $C^{1, \alpha}$ estimates 3 and restricting our estimates in small balls of definite size around $\partial \Omega$, we can assume throughout that $1-\varepsilon \leq g \leq 1+\varepsilon$ where $\varepsilon$ is as in Theorem 1.1 .

Let $y \in \Omega$ with $r:=\operatorname{dist}(y, \partial \Omega) \leq c$, for $c$ universal, and consider the maximal section $S_{\phi}(y, h)$ of $\phi$ centered at $y$, i.e.,

$$
h=\sup \left\{t \mid \quad S_{\phi}(y, t) \subset \Omega\right\} .
$$

Since $\phi$ is $C^{1,1}$ on the boundary $\partial \Omega$, by Caffarelli's strict convexity theorem, $\phi$ is strictly convex in $\Omega$. This implies the existence of the above maximal section $S_{\phi}(y, h)$ of $\phi$ centered at $y$ with $h>0$. By [5. Proposition 3.2] applied at the point $x_{0} \in \partial S_{\phi}(y, h) \cap \partial \Omega$, we have

$$
h^{1 / 2} \sim r,
$$

and $S_{\phi}(y, h)$ is equivalent to an ellipsoid $E$ i.e

$$
c E \subset S_{\phi}(y, h)-y \subset C E,
$$

where

$$
E:=h^{1 / 2} A_{h}^{-1} B_{1}, \quad \text { with } \quad\left\|A_{h}\right\|,\left\|A_{h}^{-1}\right\| \leq C|\log h| ; \operatorname{det} A_{h}=1 .
$$

We denote

$$
\phi_{y}:=\phi-\phi(y)-\nabla \phi(y)(x-y) .
$$

The rescaling $\tilde{\phi}: \tilde{S}_{1} \rightarrow \mathbb{R}$ of $u$

$$
\tilde{\phi}(\tilde{x}):=\frac{1}{h} \phi_{y}(T \tilde{x}) \quad x=T \tilde{x}:=y+h^{1 / 2} A_{h}^{-1} \tilde{x}
$$

satisfies

$$
\operatorname{det} D^{2} \tilde{\phi}(\tilde{x})=\tilde{g}(\tilde{x}):=g(T \tilde{x}),
$$

and

$$
B_{c} \subset \tilde{S}_{1} \subset B_{C}, \quad \tilde{S}_{1}=\bar{h}^{-1 / 2} A_{\bar{h}}\left(S_{y, \bar{h}}-y\right),
$$

where $\tilde{S}_{1}:=S_{\tilde{\phi}}(0,1)$ represents the section of $\tilde{\phi}$ at the origin at height 1 .

We define also the rescaling $\tilde{u}$ for $u$

$$
\tilde{u}(\tilde{x}):=h^{-1 / 2}\left(u(T \tilde{x})-u\left(x_{0}\right)-\nabla u\left(x_{0}\right)\left(T \tilde{x}-x_{0}\right)\right), \quad \tilde{x} \in \tilde{S}_{1} .
$$

Then $\tilde{u}$ solves

$$
\tilde{\Phi}^{i j} \tilde{u}_{i j}=\tilde{f}(\tilde{x}):=h^{1 / 2} f(T \tilde{x}) .
$$


Now, we apply Gutiérrez-Nguyen's interior $C^{1, \alpha}$ estimates [3] to $\tilde{u}$ to obtain

$$
\left|D \tilde{u}\left(\tilde{z}_{1}\right)-D \tilde{u}\left(\tilde{z}_{2}\right)\right| \leq C\left|\tilde{z}_{1}-\tilde{z}_{2}\right|^{\beta}\left\{\|\tilde{u}\|_{L^{\infty}\left(\tilde{S}_{1}\right)}+\|\tilde{f}\|_{L^{p}\left(\tilde{S}_{1}\right)}\right\}, \quad \forall \tilde{z}_{1}, \tilde{z}_{2} \in \tilde{S}_{1 / 2},
$$

for some small constant $\beta \in(0,1)$ depending only on $n, \lambda, \Lambda$.

By (3.17), we can decrease $\beta$ if necessary and thus we can assume that $2 \beta \leq \alpha$ where $\alpha \in(0,1)$ is the exponent in Theorem 1.1. Note that, by (3.16)

$$
\|\tilde{f}\|_{L^{p}\left(\tilde{S}_{1}\right)}=h^{1 / 2-\frac{n}{2 p}}\|f\|_{L^{p}\left(S_{y, \bar{h}}\right)} .
$$

We observe that (3.15) and (3.16) give

$$
B_{C r|\log r|}(y) \supset S_{\phi}(y, h) \supset S_{\phi}(y, h / 2) \supset B_{c_{\frac{r}{|l o g r|}}}(y)
$$

and

$$
\operatorname{diam}\left(S_{\phi}(y, h)\right) \leq C r|\log r| .
$$

By Theorem 1.1 applied to the original function $u$, (3.14) and (3.15), we have

$$
\begin{array}{r}
\|\tilde{u}\|_{L^{\infty}\left(\tilde{S}_{1}\right)} \leq C h^{-1 / 2}\left(\|u\|_{L^{\infty}(\Omega)}+\|f\|_{L^{p}(\Omega)}+\|\varphi\|_{C^{1, \gamma}(\bar{\Omega})}\right) \\
\operatorname{diam}\left(S_{\phi}(y, h)\right)^{1+\alpha} \\
\leq C r^{\alpha}|\log r|^{1+\alpha} .
\end{array}
$$

Hence, using (3.18) and the fact that $\alpha \leq 1 / 2(1-n / p)$, we get

$$
\left|D \tilde{u}\left(\tilde{z}_{1}\right)-D \tilde{u}\left(\tilde{z}_{2}\right)\right| \leq C\left|\tilde{z}_{1}-\tilde{z}_{2}\right|^{\beta} r^{\alpha}|\log r|^{1+\alpha} \forall \tilde{z}_{1}, \tilde{z}_{2} \in \tilde{S}_{1 / 2} .
$$

Rescaling back and using

$$
\tilde{z}_{1}-\tilde{z}_{2}=h^{-1 / 2} A_{h}\left(z_{1}-z_{2}\right), \quad h^{1 / 2} \sim r,
$$

and the fact that

$$
\left|\tilde{z}_{1}-\tilde{z}_{2}\right| \leq\left\|h^{-1 / 2} A_{h}\right\|\left|z_{1}-z_{2}\right| \leq C h^{-1 / 2}|\log h|\left|z_{1}-z_{2}\right| \leq C r^{-1}|\log r|\left|z_{1}-z_{2}\right|,
$$

we find

$$
\begin{aligned}
\left|D u\left(z_{1}\right)-D u\left(z_{2}\right)\right| & =\mid A_{h}\left(D \tilde{u}\left(\tilde{z}_{1}\right)-\left.D \tilde{u}\left(\tilde{z}_{2}\right)|\leq C| \log h\left|\left(r^{-1}|\log r|\left|z_{1}-z_{2}\right|\right)^{\beta} r^{\alpha}\right| \log r\right|^{1+\alpha}\right. \\
(3.19) & \leq\left|z_{1}-z_{2}\right|^{\beta} \quad \forall z_{1}, z_{2} \in S_{\phi}(y, h / 2) .
\end{aligned}
$$

Notice that this inequality holds also in the Euclidean ball $B_{c_{\frac{|l o g r|}{\mid l o}}}(y) \subset S_{\phi}(y, h / 2)$.

Combining this with Theorem 1.1, we easily obtain

$$
[D u]_{C^{\beta}(\bar{\Omega})} \leq C
$$

and the desired global $C^{1, \beta}$ bounds for $u$.

\section{ACKNOWLEDGMENTS}

The authors would like to thank the referee for constructive comments on the manuscript. The first author was partially supported by the Vietnam Institute for Advanced Study in Mathematics (VIASM), Hanoi, Vietnam. 


\section{REFERENCES}

[1] Luis A. Caffarelli, Interior a priori estimates for solutions of fully nonlinear equations, Ann. of Math. (2) 130 (1989), no. 1, 189-213, DOI 10.2307/1971480. MR.1005611 (90i:35046)

[2] Luis A. Caffarelli and Xavier Cabré, Fully nonlinear elliptic equations, American Mathematical Society Colloquium Publications, vol. 43, American Mathematical Society, Providence, RI, 1995. MR:1351007 (96h:35046)

[3] Cristian E. Gutiérrez and Truyen Nguyen, Interior gradient estimates for solutions to the linearized Monge-Ampère equation, Adv. Math. 228 (2011), no. 4, 2034-2070, DOI 10.1016/j.aim.2011.06.035. MR2836113 (2012h:35093)

[4] Nam Q. Le and Truyen Nguyen, Global $W^{2, p}$ estimates for solutions to the linearized MongeAmpère equations, Math. Ann. 358 (2014), no. 3-4, 629-700, DOI 10.1007/s00208-013-0974-6. MR3175137

[5] N. Q. Le and O. Savin, Boundary Regularity for Solutions to the Linearized Monge-Ampère Equations, Arch. Ration. Mech. Anal. 210 (2013), no. 3, 813-836, DOI 10.1007/s00205-0130653-5. MR 3116005

[6] Savin, O. A localization property at the boundary for the Monge-Ampère equation. Advances in Geometric Analysis, 45-68, Adv. Lect. Math. (ALM), 21, Int. Press, Somerville, MA, 2012.

[7] O. Savin, Pointwise $C^{2, \alpha}$ estimates at the boundary for the Monge-Ampère equation, J. Amer. Math. Soc. 26 (2013), no. 1, 63-99, DOI 10.1090/S0894-0347-2012-00747-4. MR2983006

[8] O. Savin, Global $W^{2, p}$ estimates for the Monge-Ampère equation, Proc. Amer. Math. Soc. 141 (2013), no. 10, 3573-3578, DOI 10.1090/S0002-9939-2013-11748-X. MR3080179

[9] N. N. Ural'tseva, Hölder continuity of gradients of solutions of parabolic equations with boundary conditions of Signorini type (Russian), Dokl. Akad. Nauk SSSR 280 (1985), no. 3, 563565. MR775926 (87b:35025)

[10] N. N. Ural'tseva, Estimates of derivatives of solutions of elliptic and parabolic inequalities, (Berkeley, Calif., 1986), Amer. Math. Soc., Providence, RI, 1987, pp. 1143-1149. MR934318 (90a:35033)

[11] Xu-Jia Wang, Some counterexamples to the regularity of Monge-Ampère equations, Proc. Amer. Math. Soc. 123 (1995), no. 3, 841-845, DOI 10.2307/2160809. MR.1223269 (95d:35025)

[12] Xu-Jia Wang, Schauder estimates for elliptic and parabolic equations, Chinese Ann. Math. Ser. B 27 (2006), no. 6, 637-642, DOI 10.1007/s11401-006-0142-3. MR.2273802(2007f:35106)

Department of Mathematics, Columbia University, New York, New York 10027 Current address: Institute of Mathematics, Vietnam Academy of Science and Technology, 18 Hoang Quoc Viet, 10307 Hanoi, Vietnam

Current address: Department of Mathematics, Indiana University, Bloomington, IN 47405

E-mail address: nqle@indiana.edu

Department of Mathematics, Columbia University, New York, New York 10027

E-mail address: savin@math.columbia.edu 